\title{
Analysis of active control of noise in duct by means of impedance
}

\author{
Josuke Okda, Tsuyoshi Usagawa, and Masanao Ebata \\ Faculty of Engineering, Kumamoto University, \\ 2-39-1, Kurokami, Kumamoto, 860 Japan
}

(Received 16 August 1986)

\begin{abstract}
In this paper, the principle of the active control of noise in duct is analyzed by means of impedance. It is shown that the principle is equivalent to making a short circuit on a transmission line by the equivalent impedance of the active system. From the result of the analysis, general equations and their equivalent circuits of multi-microphone and multi-loudspeaker system are deduced, and also general requirements for control without acoustical feedback which makes the system unstable, are made clear. For the practical use, some suitable combinations of microphones and loudspeakers which control the noise without acoustical feedback are shown. In addition, an electrical method by which the acoustical feedback is canceled is proposed.
\end{abstract}

PACS number: 43. 50. Gf

\section{INTRODUCTION}

Since Paul Leug ${ }^{1)}$ proposed the concept of active control of sound wave using the antiphase signal in 1934, a number of reports based on his idea have been published in this field. Recently the researches in the active noise control have become more and more extensive, but almost no one has succeeded in perfect control of noise over wide frequency range based on this principle and few method have been put to practical use.

It is difficult to analyze the active control system strictly only on the principle of noise reduction by the wave with an antiphase of the original one. Then we should reexamine the basic principle of active control.

In this paper, the principle of active control of noise in duct is analyzed by means of impedance. And suitable configurations of system and the requirements for control without acoustic feedback are shown.

\section{PRINCIPLE AND METHODS OF ACTIVE CONTROL OF NOISE IN DUCT}

\subsection{Principle}

When noise in duct travels down to the outlet of the duct where noise is radiated into the room, the radiated noise poses a problem. In order to suppress a travelling sound in duct, an electro-acoustic transducer (loudspeaker) is mounted on the duct wall as shown in Fig. 1. Assume that the walls of the duct are frictionless, the sound field in the duct is onedimensional and the effective diameter of the transducer diaphragm is small compared with the wave length.

The notation used in the figure is as follows: $Z$ is the characteristic impedance of the duct, $R_{\mathrm{r}}$ is the radiation resistance of the outlet of the duct, $z_{\mathrm{s}}$ is the acoustic impedance of the transducer, $p_{\mathrm{s}}$ is the driving force of the transducer which is represented by sound pressure, and $l_{\mathrm{a}}$ is the equivalent length between the transducer and the outlet of the duct which includes the radiation reactance of the duct. $z_{\mathrm{s}}$ is the mechanical impedance of the transducer divided by 


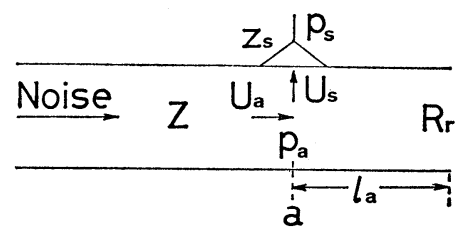

Fig. 1 System configuration.

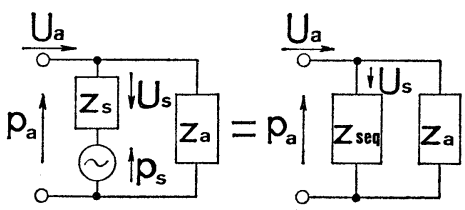

Fig. 2 Equivalent circuit.

$S_{\mathrm{s}}{ }^{2}$ and $p_{\mathrm{s}}$ is the driving force divided by $S_{\mathrm{s}}$, which is the effective area of the transducer diaphragm. The impedance looking back into the outlet of the duct from the plane " $a$ " where the transducer is mounted, is

$$
z_{\mathrm{a}}=R_{\mathrm{a}}+j X_{\mathrm{a}}=Z \frac{R_{\mathrm{r}} \cos k l_{\mathrm{a}}+j Z \sin k l_{\mathrm{a}}}{Z \cos k l_{\mathrm{a}}+j R_{\mathrm{r}} \sin k l_{\mathrm{a}}}
$$

where $k$ is the wave number.

Let $p_{\mathrm{a}}$ be the sound pressure at "a," and let $U_{\mathrm{a}}$ and $U_{\mathrm{s}}$ be the volume velocity flowing into "a" from upstream duct and that flowing into the transducer respectively. The equivalent circuit is shown in Fig. 2 where $z_{\mathrm{s} \text { eq }}$ is the equivalent impedance, that is

$$
z_{\mathrm{s} \mathrm{eq}}=\frac{p_{\mathrm{a}}}{U_{\mathrm{s}}}
$$

The reflection coefficient of sound pressure at " $a$ " is given as follows:

$$
\Gamma=\frac{\frac{z_{\mathrm{seq}} z_{\mathrm{a}}}{z_{\mathrm{s} \text { eq }}+z_{\mathrm{a}}}-Z}{\frac{z_{\mathrm{seq}} z_{\mathrm{a}}}{z_{\mathrm{s} \text { eq }}+z_{\mathrm{a}}}+Z}=\frac{z_{\mathrm{seq}}\left(z_{\mathrm{a}}-Z\right)-z_{\mathrm{a}} Z}{z_{\mathrm{seq}}\left(z_{\mathrm{a}}+Z\right)+z_{\mathrm{a}} Z}
$$

When the pressure of the incident progressive sound wave at the plane "a" is $p_{\text {in }}$ and the reflecting one is $p_{\mathrm{r}}, p_{\mathrm{a}}$ is represented by the following equation.

$$
p_{\mathrm{a}}=p_{\text {in }}+p_{\mathrm{r}}=\frac{2 z_{\mathrm{seq}} z_{\mathrm{a}}}{z_{\mathrm{seq}}\left(z_{\mathrm{a}}+Z\right)+z_{\mathrm{a}} Z} p_{\text {in }}
$$

And the travelling sound power transmitted downward is

$$
W=\left|\frac{p_{\mathrm{a}}}{z_{\mathrm{a}}}\right|^{2} R_{\mathrm{a}}
$$

When $p_{\mathrm{s}}=0, z_{\mathrm{s} \text { eq }}=z_{\mathrm{s}}$ and the pressure at " $\mathrm{a}$ " is

$$
p_{\mathrm{a} 0}=\frac{2 z_{\mathrm{s}} z_{\mathrm{a}}}{z_{\mathrm{s}}\left(z_{\mathrm{a}}+Z\right)+z_{\mathrm{a}} Z} p_{\mathrm{in}}
$$

and

$$
p_{\mathrm{a}}=p_{\mathrm{a} 0}+p_{\mathrm{as}}
$$

where $p_{\text {as }}$ is the sound pressure radiated from the transducer.

When the transducer is not mounted on the wall, $z_{\mathrm{s}}$ is infinite and the pressure at " $\mathrm{a}$ " is given as

$$
p_{\mathrm{a} 00}=\frac{2 z_{\mathrm{a}}}{z_{\mathrm{a}}+Z} p_{\text {in }}
$$

and the sound power transmitted downstream is

$$
W_{00}=\left|\frac{p_{\mathrm{a} 00}}{z_{\mathrm{a}}}\right|^{2} R_{\mathrm{a}}
$$

The insertion loss due to the second source, the transducer system, is described as

$$
\begin{aligned}
I L & =10 \log \frac{W_{00}}{W}=20 \log \left|\frac{p_{\mathrm{a} 00}}{p_{\mathrm{a}}}\right| \\
& =20 \log \left|1+\frac{z_{\mathrm{a}} Z}{z_{\mathrm{seq}}\left(z_{\mathrm{a}}+Z\right)}\right|
\end{aligned}
$$

If $I L$ is positive, the sound power travelling downstream, namely the power radiating from the outlet of the duct, could be reduced. Especially, if $p_{\mathrm{a}}=0$, $I L$ could be infinite. The condition: $p_{\mathrm{a}}=0$, means that $p_{\mathrm{as}}=-p_{\mathrm{a} 0}$. As shown by P. Leug, the principle of the active control of noise is to cancel the noise by the addition of the antiphase noise using the second source. It must be noticed, however, that the sound pressure to be canceled is not $p_{\text {a } 00}$ which is one before the transducer is mounted but $p_{\mathrm{a} 0}$ which is one after the transducer without driving force is mounted.

Viewed from a different standpoint, making $p_{\mathrm{a}}$ equal zero or $I L$ equal infinite, is making the equivalent impedance $z_{\mathrm{s} \text { eq }}$ equal zero. In other words, the principle of the active control of noise in duct is to make short-circuit on the transmition line. If $z_{s \text { eq }}$ can not be perfectly equal to zero, the characteristics of $I L$ are influenced by the characteristics of $z_{\mathrm{s} \text { eq }}$ and $z_{\mathrm{a}}$.

When $z_{\mathrm{s} \text { eq }}$ is not the impedance of an active system but that of a resonator, it becomes the resonatortype muffler. The resonator-type muffler makes a short-circuit in the duct at the frequency of resonance.

\subsection{Methods of Active Control}

There are three methods by which the input signal to the transducer is picked out, ${ }^{2)}$ that is,

(1) independent electric source.

(2) sound pressure in the field. 


\section{J. OKDA et al.: ANALYSIS OF ACTIVE CONTROL OF NOISE IN DUCT}

(3) vibration of the moving part of the transducer (motional feedback).

On the first method, the electric signal for control can not follow the change of the boundary condition. T. Kosaka and S. Yamada ${ }^{3)}$ controlled the fundamental frequency of the second source on the basis of the mechanical vibration of the noise source and also controlled the amplitude and the phase of harmonics on the basis of the residual noise detected by a microphone. The second method is the most popular one. In the third method, any microphones are not required, so that the system can be used in severe condition such as in high temperature, or in ducts with a high speed air current, or with a dusty current.4)

In case where a microphone is used, the acoustic feedback from the loudspeaker to the microphone becomes a serious problem. To reduce the feedback, various systems with multiple microphones or multiple loudspeakers have been proposed. ${ }^{5-12)}$ But in these systems, the impedances $z_{\mathrm{s}}$ and $z_{\mathrm{a}}$ were neglected. As mentioned above, $z_{\mathrm{s}}$ and $z_{\mathrm{a}}$ can not be neglected compared with $Z$. To control the noise over the wide frequency range, it is necessary to take $z_{\mathrm{s}}$ and $z_{\mathrm{a}}$ into account.

\section{MULTI-MICROPHONE AND MULTI-LOUDSPEAKER SYSTEM}

\subsection{Configuration of System and Assumptions}

Assumptions in the analysis are as follows:

a) The duct is frictionless and the sound field in the duct is one dimensional.

b) All microphones have the same characteristics and they do not disturb the sound field because of their small size and high impedance.

c) All loudspeakers have the same characteristics and the effective diameter of their diaphragm is sufficiently small compared with the wavelength.

The configuration of the system is shown in Fig. 3 where the characteristic impedance of the duct is $Z$ and the acoustic impedance of the moving part of

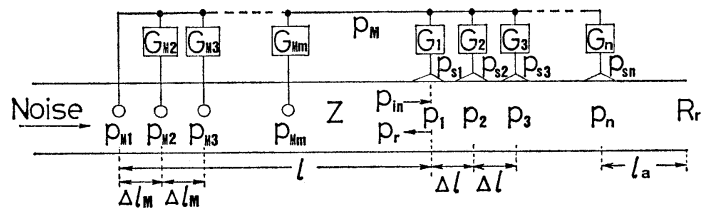

Fig. 3 Configuration of system with $m$ microphones and $n$ loudspeakers. the loudspeaker which includes the motional impedance is $z_{\mathrm{s}} . \quad m$ microphones are mounted in the duct where $\Delta l_{\mathrm{M}}$ is the spacing of each microphone. Let the sound pressure at the $i$-th microphone be $p_{\mathrm{M} i}$ $(i=1,2, \ldots, m)$. The gain and the phase of the output of each microphone are controlled by a network with transfer function $G_{\mathrm{M} i}$ where $G_{\mathrm{M} 1}=1$, and then they are added up. As shown in Fig. 3, this output is denoted by the sound pressure $p_{\mathrm{M}}$ which is represented in terms of at $p_{\mathrm{M} 1}$ and it is supplied to each loudspeaker through the power amplifier $G_{i}(i=$ $1,2, \ldots, n) . \quad n$ loudspeakers are also mounted on the duct wall where the spacing is $\Delta l$. The distance between the first microphone and the first loudspeaker is $l$. Let $z_{\mathrm{a}}$ be the impedance looking back into the outlet of the duct from the $n$-th loudspeaker. Sound pressures and volume velocities are defined as follows:

$p_{i}$ : the sound pressure at the $i$-th loudspeaker.

$p_{\mathrm{s} i}$ : the driving forth through $G_{i}$ which is represented in terms of sound pressure.

$U_{i}:$ the volume velocity flowing into the section of the $i$-th loudspeaker, this is divided to $U_{\mathrm{s} i}$ and volume velocity going down to the outlet.

$U_{\mathrm{s} i}$ : the volume velocity flowing into the $i$-th loudspeaker.

$p_{\text {in }}$ : the pressure of incident progressive sound wave flowing in the section of the first loudspeaker.

$p_{\mathrm{r}}$ : the pressure of the progressive sound wave reflected at the section of the first loudspeaker.

$$
p_{1}=p_{\text {in }}+p_{\mathrm{r}}, \quad Z U_{1}=p_{\text {in }}-p_{\mathrm{r}}
$$

\subsection{Loudspeaker System}

Figure 4 shows the section of the duct on which the $i$-th and the $(i+1)$-th loudspeakers are mounted with $\Delta l$ spacing and its equivalent circuit.

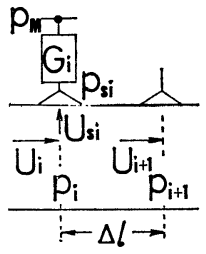

(a)

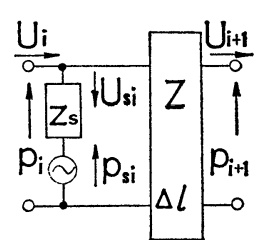

(b)
Fig. 4 Equivalent circuit of $i$-th section, (a) configuration of $i$-th section (b) equivalent circuit. 


$$
\begin{gathered}
p_{i+1}=\cos x \cdot p_{i}-j Z \sin x \cdot\left(U_{i}-U_{\mathrm{s} i}\right) \\
U_{i+1}=-j \frac{1}{Z} \sin x \cdot p_{i}+\cos x \cdot\left(U_{i}-U_{\mathrm{s} i}\right) \\
p_{i}=p_{\mathrm{s} i}+z_{\mathrm{s}} U_{\mathrm{s} i} \\
p_{\mathrm{s} i}=G_{i} p_{\mathrm{M}}
\end{gathered}
$$

where

$$
x=k \Delta l
$$

From these equations

$$
\begin{aligned}
& p_{i+1}=A p_{i}-B U_{i}-\frac{1}{z_{\mathrm{s}}} B G_{i} p_{\mathrm{M}} \\
& U_{i+1}=-C p_{i}+D U_{i}+\frac{1}{z_{\mathrm{s}}} D G_{i} p_{\mathrm{M}}
\end{aligned}
$$

where

$$
\left.\begin{array}{l}
A=\cos x+j \frac{Z}{z_{\mathrm{s}}} \sin x, \quad B=j Z \sin x \\
C=j \frac{1}{Z}\left(\sin x-j \frac{Z}{z_{\mathrm{s}}} \cos x\right), \quad D=\cos x
\end{array}\right\}
$$

For $p_{n}$

$$
\begin{aligned}
& p_{n}=p_{\mathrm{s} n}+z_{\mathrm{s}} U_{\mathrm{s} n} \\
& p_{n}=z_{\mathrm{a}}\left(U_{n}-U_{\mathrm{s} n}\right)
\end{aligned}
$$

$p_{n}$ can be described as following two equations, using $p_{\text {in }}$ and $p_{\mathrm{r}}$

$$
p_{n}=\left(A_{n}-\frac{B_{n}}{Z}\right) p_{\text {in }}+\left(A_{n}+\frac{B_{n}}{Z}\right) p_{\mathrm{r}}-G_{\mathrm{B}_{n}} p_{\mathrm{M}}
$$

and

$$
\begin{aligned}
\left(\frac{Z}{z_{\mathrm{s}}}+\frac{Z}{z_{\mathrm{a}}}\right) p_{n}= & \left(D_{n}-Z C_{n}\right) p_{\mathrm{in}} \\
& -\left(D_{n}+Z C_{n}\right) p_{\mathrm{r}}+G_{\mathrm{D}_{n}} p_{\mathrm{M}}
\end{aligned}
$$

where

$$
\begin{aligned}
& A_{i}=A \cdot A_{i-1}+B \cdot C_{i-1} \\
& B_{i}=A \cdot B_{i-1}+B \cdot D_{i-1} \\
& C_{i}=C \cdot A_{i-1}+D \cdot C_{i-1} \\
& D_{i}=C \cdot B_{i-1}+D \cdot D_{i-1} \\
& A_{i} D_{i}-B_{i} C_{i}=1 \\
& A_{1}=D_{1}=1, \quad B_{1}=C_{1}=0 \\
& A_{2}=A=\cos x+j \frac{Z}{z_{\mathrm{s}}} \sin x, \quad B_{2}=B=j Z \sin x \\
& \left.C_{2}=C=j \frac{1}{Z}\left(\sin x-j \frac{Z}{z_{\mathrm{s}}} \cos x\right), \quad D_{2}=D=\cos x\right)
\end{aligned}
$$

$$
\left.\begin{array}{rl}
G_{\mathrm{B}_{n}} & =\frac{1}{z_{\mathrm{s}}} \sum_{i=1}^{n} B_{n+1-i} G_{i} \\
G_{\mathrm{D}_{n}} & =\frac{Z}{z_{\mathrm{s}}} \sum_{i=1}^{n} D_{n+1-i} G_{i}
\end{array}\right\}
$$

\subsection{Microphone System}

The sound pressure at each microphone is given as

$$
p_{\mathrm{M} i}=\epsilon^{j k l} \epsilon^{-j(i-1) y} p_{\mathrm{in}}+\epsilon^{-j k l} \epsilon^{j(i-1) y} p_{\mathrm{r}}
$$

where

$$
y=k \Delta l_{\mathrm{M}}
$$

hence the output of the microphone system which is represented in terms of sound pressure is given as

$$
p_{\mathrm{M}}=\epsilon^{j k l} G_{\mathrm{im}} p_{\mathrm{in}}+\epsilon^{-j k l} G_{\mathrm{rm}} p_{\mathrm{r}}
$$

where

$$
\left.\begin{array}{l}
G_{\mathrm{im}}=\sum_{i=1}^{m} \epsilon^{-j(i-1) y} G_{\mathrm{M} i} \\
G_{\mathrm{rm}}=\sum_{i=1}^{m} \epsilon^{j(i-1) y} G_{\mathrm{M} i}
\end{array}\right\}
$$

3.4 General Equation of Total System and Its Equivalent Circuit

From Eq. (30), Eqs. (22) and (23) yield to the equations as follows:

$$
\begin{gathered}
p_{n}=\left\{\left(A_{n}-\frac{B_{n}}{Z}\right)-\epsilon^{j k l} G_{\mathrm{im}} G_{\mathrm{B}_{n}}\right\} p_{\mathrm{in}} \\
+\left\{\left(A_{n}+\frac{B_{n}}{Z}\right)-\epsilon^{-j k l} G_{\mathrm{rm}} G_{\mathrm{B}_{n}}\right\} p_{\mathrm{r}} \\
\left(\frac{Z}{z_{\mathrm{s}}}+\frac{Z}{z_{\mathrm{a}}}\right) p_{n}=\left\{\left(D_{n}-Z C_{n}\right)+\epsilon^{j k l} G_{\mathrm{im}} G_{\mathrm{D}_{n}}\right\} p_{\mathrm{in}} \\
-\left\{\left(D_{n}+Z C_{n}\right)-\epsilon^{-j k l} G_{\mathrm{rm}} G_{\mathrm{D} n}\right\} p_{\mathrm{r}}
\end{gathered}
$$

hence

$$
\begin{aligned}
\frac{\Delta p_{n}}{p_{\text {in }}}= & 2+\left\{\left(A_{n}+\frac{B_{n}}{Z}\right) \epsilon^{j k l} G_{\mathrm{im}}\right. \\
& \left.-\left(A_{n}-\frac{B_{n}}{Z}\right) \epsilon^{-j k l} G_{\mathrm{rm}}\right\} G_{\mathrm{D}_{n}} \\
& -\left\{\left(D_{n}+Z C_{n}\right)+\epsilon^{j k l} G_{\mathrm{im}}\right. \\
& \left.+\left(D_{n}-Z C_{n}\right) \epsilon^{-j k l} G_{\mathrm{rm}}\right\} G_{\mathrm{B}_{n}} \\
\frac{\Delta p_{\mathrm{r}}}{p_{\mathrm{in}}}= & \left\{\left(D_{n}-Z C_{n}\right)-\left(\frac{Z}{z_{\mathrm{s}}}+\frac{Z}{z_{\mathrm{a}}}\right)\left(A_{n}-\frac{B_{n}}{Z}\right)\right\} \\
& +\epsilon^{j k l} G_{\mathrm{im}}\left\{G_{\mathrm{D}_{n}}+\left(\frac{Z}{z_{\mathrm{s}}}+\frac{Z}{z_{\mathrm{a}}}\right) G_{\mathrm{B}_{n}}\right\}
\end{aligned}
$$




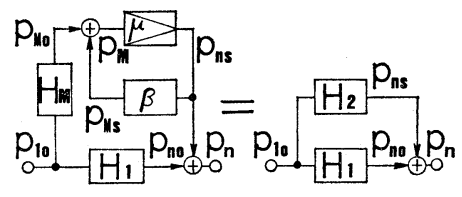

Fig. 5 Block diagram for total system.

$$
\begin{aligned}
\Delta= & \left\{\left(D_{n}+Z C_{n}\right)+\left(\frac{Z}{z_{\mathrm{s}}}+\frac{Z}{z_{\mathrm{a}}}\right)\left(A_{n}+\frac{B_{n}}{Z}\right)\right\} \\
& -\epsilon^{j k l} G_{\mathrm{rm}}\left\{G_{\mathrm{D}_{n}}+\left(\frac{Z}{z_{\mathrm{s}}}+\frac{Z}{z_{\mathrm{a}}}\right) G_{\mathrm{B}_{n}}\right\}
\end{aligned}
$$

The sound pressures $p_{\mathrm{n}}, p_{\mathrm{r}}$ and $p_{\mathrm{M}}$ are represented by $p_{\mathrm{n} 0}, p_{\mathrm{r} 0}$ and $p_{\mathrm{M} 0}$ which are the pressure when $p_{\mathrm{s} i}=$ $0\left(G_{\mathrm{B} i}=G_{\mathrm{D} i}=0\right)$, and $p_{\mathrm{ns}}, p_{\mathrm{rs}}$ and $p_{\mathrm{Ms}}$ which are produced by $p_{\mathrm{s} i}$.

$$
p_{\mathrm{n}}=p_{\mathrm{n} 0}+p_{\mathrm{ns}}, \quad p_{\mathrm{r}}=p_{\mathrm{r} 0}+p_{\mathrm{rs}}, \quad p_{\mathrm{M}}=p_{\mathrm{M} 0}+p_{\mathrm{Ms}}
$$

The block diagram which shows the operation can be represented in Fig. 5. Where

$$
\left.\begin{array}{l}
H_{1}=\frac{p_{\mathrm{n} 0}}{p_{10}}, H_{\mathrm{M}}=\frac{p_{\mathrm{M} 0}}{p_{10}}, \mu=\frac{p_{\mathrm{ns}}}{p_{\mathrm{M}}}, \beta=\frac{p_{\mathrm{Ms}}}{p_{\mathrm{ns}}} \\
H_{2}=H_{\mathrm{M}} \frac{\mu}{1-\mu \beta}=\frac{p_{\mathrm{ns}}}{p_{10}}
\end{array}\right\}
$$

3.5 Requirement for Control Attended with no Feedback

Substitution of Eq. (37) in Eq. (35) results in the equation as follows:

$$
\begin{aligned}
\frac{p_{\text {rn }}}{p_{\text {in }}}= & \frac{G_{\mathrm{D}_{n}}+\left(\frac{Z}{z_{\mathrm{s}}}+\frac{Z}{z_{\mathrm{a}}}\right) G_{\mathrm{B}_{n}}}{\Delta\left\{\left(D_{n}+Z C_{n}\right)+\left(\frac{Z}{z_{\mathrm{s}}}+\frac{Z}{z_{\mathrm{a}}}\right)\left(A_{n}+\frac{B_{n}}{Z}\right)\right\}} \\
& \times\left[\left\{\left(D_{n}+Z C_{n}\right)+\left(\frac{Z}{z_{\mathrm{s}}}+\frac{Z}{z_{\mathrm{a}}}\right)\right.\right. \\
& \left.\cdot\left(A_{n}+\frac{B_{n}}{Z}\right)\right\} \epsilon^{j k l} G_{\mathrm{im}} \\
& +\left\{\left(D_{n}-Z C_{n}\right)-\left(\frac{Z}{z_{\mathrm{s}}}+\frac{Z}{z_{\mathrm{a}}}\right)\right. \\
& \left.\left.\cdot\left(A_{n}-\frac{B_{n}}{Z}\right)\right\} \epsilon^{-j k l} G_{\mathrm{rm}}\right]
\end{aligned}
$$

Substitution of Eq. (37) in Eq. (30) results in the equation as follows:

$$
p_{\mathrm{Ms}}=\epsilon^{-j k l} G_{\mathrm{rm}} \cdot p_{\mathrm{rs}}
$$

The condition in which there is no feedback $(\beta=$ 0 ) is given by the following equation.

$$
G_{\mathrm{rm}}=0
$$

or

$$
G_{\mathrm{D}_{n}}+\left(\frac{Z}{z_{\mathrm{s}}}+\frac{Z}{z_{\mathrm{a}}}\right) G_{\mathrm{B}_{n}}=0
$$

When $n=1$, the condition of complete cancelation $\left(p_{1}=0\right)$ is given as follows:

$$
\frac{Z}{z_{\mathrm{s}}} G_{1}=-\frac{2}{\epsilon^{j k l} G_{\mathrm{im}}-\epsilon^{-j k l} G_{\mathrm{rm}}}
$$

When $n \geq 2$, the condition of complete cancelation corresponding to Eq. (41) can be represented by the equation as follows:

$$
\left(A_{n}+\frac{B_{n}}{Z}\right) G_{\mathrm{D}_{n}}-\left(D_{n}+Z C_{n}\right) G_{\mathrm{B}_{n}}=-\frac{2 \epsilon^{-j k l}}{G_{\mathrm{im}}}
$$

and the equation corresponding to Eq. (42), is represented by the following equation,

$$
\begin{aligned}
\frac{2}{G_{\mathrm{B}_{n}}}= & \left\{\left(D_{n}+Z C_{n}\right)+\left(\frac{Z}{z_{\mathrm{s}}}+\frac{Z}{z_{\mathrm{a}}}\right)\right. \\
& \left.\cdot\left(A_{n}+\frac{B_{n}}{Z}\right)\right\} \epsilon^{j k l} G_{\mathrm{im}} \\
& +\left\{\left(D_{n}-Z C_{n}\right)-\left(\frac{Z}{z_{\mathrm{s}}}+\frac{Z}{z_{\mathrm{a}}}\right)\right. \\
& \left.\cdot\left(A_{n}-\frac{B_{n}}{Z}\right)\right\} \epsilon^{-j k l} G_{\mathrm{rm}}
\end{aligned}
$$

3.6 Input Acoustic Impedance of the Loudspeaker System

Substituting Eq. (11) and Eq. (35) in $z_{\text {in }}=p_{1} / U_{1}$ that is the input acoustic impedance of the loudspeaker system; $z_{\text {in }}$ results in the equation as follows:

$$
\begin{aligned}
z_{\mathrm{in}}= & Z\left[2\left\{D_{n}+\left(\frac{Z}{z_{\mathrm{s}}}+\frac{Z}{z_{\mathrm{a}}}\right) \frac{B_{n}}{Z}\right\}\right. \\
& +\left(\epsilon^{j k l} G_{\mathrm{im}}+\epsilon^{-j k l} G_{\mathrm{rm}}\right) \\
& \left.+\left\{G_{\mathrm{D}_{n}}+\left(\frac{Z}{z_{\mathrm{s}}}+\frac{Z}{z_{\mathrm{a}}}\right) G_{\mathrm{B}_{n}}\right\}\right] \\
& /\left[2\left\{Z C_{n}+\left(\frac{Z}{z_{\mathrm{s}}}+\frac{Z}{z_{\mathrm{a}}}\right) A_{n}\right\}\right. \\
& +\left(\epsilon^{j k l} G_{\mathrm{im}}-\epsilon^{-j k l} G_{\mathrm{rm}}\right) \\
& \left.+\left\{G_{\mathrm{D}_{n}}+\left(\frac{Z}{z_{\mathrm{s}}}+\frac{Z}{z_{\mathrm{a}}}\right) G_{\mathrm{B}_{n}}\right\}\right]
\end{aligned}
$$

Especially when $n=1$ and $P_{1}=0$,

$$
z_{\text {in }}=0
$$

In this case, the radiating sound power from the original noise source becomes only reactive one.

For $n \geq 2, z_{\text {in }} \neq 0$ in general and

$$
z_{\text {in }}=R_{\text {in }}+j X_{\text {in }}
$$


The power radiating from the original noise source is represented by the following equation.

$$
W=\left|U_{1}\right|^{2} R_{\text {in }}
$$

When $p_{n}=0$, the power $W$ is consumed by each loudspeaker except the $n$-th one. Namely

$$
W=\sum_{i=1}^{n-1}\left|U_{\mathrm{s} i}\right|^{2} R_{\mathrm{s}}
$$

where

$$
z_{\mathrm{s}}=R_{\mathrm{s}}+j X_{\mathrm{s}}
$$

Especially, when the condition Eq. (42) is valid

$$
z_{\text {in }}=Z \frac{D_{n}+\left(\frac{Z}{z_{\mathrm{s}}}+\frac{Z}{z_{\mathrm{a}}}\right) \frac{B_{n}}{Z}}{Z C_{n}+\left(\frac{Z}{z_{\mathrm{s}}}+\frac{Z}{z_{\mathrm{a}}}\right) A_{n}}
$$

In this case, $z_{\text {in }}$ is equal to the input impedance at $p_{\mathrm{s} i}=0$.

\section{ARRANGEMENT OF MICROPHONE AND LOUDSPEAKER}

There are two conditions to cancel the sound in the duct, that is $\beta=0$ and $p_{n}=0$, so that only two variables are required. As $G_{\mathrm{M} 1}=1$, the numbers $m$ and $n$ must satisfy the equation; $m+n=3$, that is $m=1$ and $n=2$ or $m=2$ and $n=1$. The arrangement of microphones and loudspeakers are shown in Fig. 6 in which those of $m=n=1$ are also shown.

\section{1 $m=n=1$}

In this case, the equation of loudspeaker system; Eq. (23) is reduced to the equation as follows:

$$
\left(\frac{Z}{z_{\mathrm{s}}}+\frac{Z}{z_{\mathrm{a}}}\right) p_{1}=p_{\mathrm{in}}-p_{\mathrm{r}}+\frac{Z}{z_{\mathrm{s}}} G_{1} p_{\mathrm{M}}
$$

a) In case where a microphone is above a loudspeaker, as shown in Fig. 6 (a)

$p_{\mathrm{M}}$ is given as

$$
p_{\mathrm{M}}=\epsilon^{j k l} p_{\text {in }}+\epsilon^{-j k l} p_{\mathrm{r}}
$$

hence

$$
\begin{gathered}
\frac{p_{1}}{p_{\mathrm{in}}}=\frac{2\left(1+j \frac{Z}{z_{\mathrm{s}}} \sin k l \cdot G_{1}\right)}{\left(1+\frac{Z}{z_{\mathrm{s}}}+\frac{Z}{z_{\mathrm{a}}}\right)-\epsilon^{-j k l} \frac{Z}{z_{\mathrm{a}}} G_{1}} \\
\beta=\frac{p_{\mathrm{Ms}}}{p_{1 \mathrm{~s}}}=\epsilon^{-j k l} \\
\mu \beta=\frac{p_{\mathrm{Ms}}}{p_{\mathrm{M}}}=\frac{Z}{z_{\mathrm{s}}} G_{1} \frac{\epsilon^{-j k l}}{1+\frac{Z}{z_{\mathrm{s}}}+\frac{Z}{z_{\mathrm{a}}}}
\end{gathered}
$$

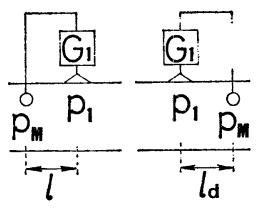

(a)

(b)
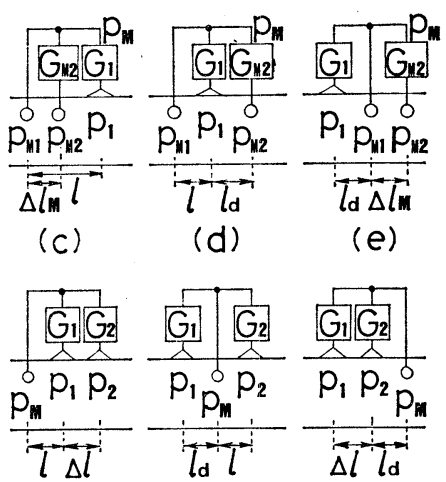

(f) (g)

(h)

Fig. 6 Arrangements of microphones and loudspeakers.

When $\mu \beta=1$, the system becomes unstable. Therefore, in order that the system is stable

$$
G_{1} \neq\left(1+\frac{z_{\mathrm{s}}}{Z}+\frac{z_{\mathrm{s}}}{z_{\mathrm{a}}}\right) \epsilon^{j k l}
$$

In order that $p_{1}=0, G_{1}$ must be given by the equation as follows:

$$
G_{1}=-\frac{1}{j \frac{Z}{z_{\mathrm{s}}} \sin k l}
$$

If the microphone is mounted very near the loudspeaker, $l=0$, then Eqs. (56), (58) and (59) reduced to the following equations.

$$
\begin{gathered}
\beta=1 \\
G_{1} \neq\left(1+\frac{z_{\mathrm{s}}}{Z}+\frac{z_{\mathrm{s}}}{z_{\mathrm{a}}}\right) \\
G_{1}=-\frac{1}{j \frac{Z}{z_{\mathrm{s}}}(k l)_{l \rightarrow 0}}
\end{gathered}
$$

In this case, the characteristics required for $G_{1}$ seems to become simple. But the sound field near the loudspeaker can not considered as an one-dimensional one in the actual condition, so that some corrections are required in Eqs. $(60) \sim(62)$ and they are not simple. When the microphone is mounted very near the loudspeaker, the condition is always the same as one 


\section{J. OKDA et al.: ANALYSIS OF ACTIVE CONTROL OF NOISE IN DUCT}

mentioned above.

b) In case where a loudspeaker is mounted above a microphone, as shown in Fig. 6 (b)

$p_{\mathrm{M}}$ is given as

$$
p_{\mathrm{M}}=\left(\cos k l_{\mathrm{d}}-j \frac{Z}{z_{\mathrm{a}}} \sin k l_{\mathrm{d}}\right) p_{1}
$$

hence

$$
\begin{gathered}
\frac{p_{1}}{p_{\text {in }}}=\frac{2}{\left(1+\frac{Z}{z_{\mathrm{s}}}+\frac{Z}{z_{\mathrm{a}}}\right)-\left(\cos k l_{\mathrm{d}}-j \frac{Z}{z_{\mathrm{a}}} \sin k l_{\mathrm{d}}\right) \frac{Z}{z_{\mathrm{s}}} G_{1}} \\
\beta=\cos k l_{\mathrm{d}}-j \frac{Z}{z_{\mathrm{a}}} \sin k l_{\mathrm{d}}
\end{gathered}
$$

In order that the system is stable

$$
G_{1} \neq \frac{1+\frac{z_{\mathrm{s}}}{Z}+\frac{z_{\mathrm{s}}}{z_{\mathrm{a}}}}{\cos k l_{\mathrm{d}}-j \frac{Z}{z_{\mathrm{a}}} \sin k l_{\mathrm{d}}}
$$

and in order that $p_{1}=0, G_{1}$ must be infinite.

$$
G_{1} \rightarrow \infty
$$

In the system of one microphone and one loudspeaker, the frequency range of noise control must be limited in a very narrow one by the reason of the restriction; $\mu \beta \neq 1$.

If $p_{1} \neq 0, I L$ is influenced by $z_{\mathrm{s}}$ and $z_{\mathrm{a}}$ as expressed in Chap. 2.

\section{$4.2 m=2, n=1$}

There are three cases.

c) In case where the system is shown in Fig. 6 (c) From Eq. (41) the condition of $\beta=0$ is given by the following equation.

$$
G_{\mathrm{M} 1}=-\epsilon^{-j k \Delta l_{\mathrm{M}}}
$$

and from Eq. (43) it follows that in order for $p_{1}$ to be zero,

$$
G_{1}=-\frac{\epsilon^{-j k\left(l-\Delta l_{\mathrm{M}}\right)}}{j \frac{Z}{z_{\mathrm{s}}} \sin k \Delta l}
$$

d) In case where the system is shown in Fig. 6 (d)

The sound pressure at each microphone is given as

$$
\begin{gathered}
p_{\mathrm{M} 1}=\epsilon^{j k l} p_{\mathrm{in}}+\epsilon^{-j k l} p_{\mathrm{r}} \\
p_{\mathrm{M} 2}=\left(\cos k l_{\mathrm{d}}-j \frac{Z}{z_{\mathrm{a}}} \sin k l_{\mathrm{d}}\right) p_{1}
\end{gathered}
$$

hence

$$
\begin{aligned}
\frac{p_{1}}{p_{\text {in }}}= & 2\left(1+j \frac{Z}{z_{\mathrm{s}}} \sin k l \cdot G_{1}\right) \\
& /\left[\left(1+\frac{Z}{z_{\mathrm{s}}}+\frac{Z}{z_{\mathrm{a}}}\right) \epsilon^{j k l}\right. \\
& \left.-\left\{1+\left(\cos k l_{\mathrm{d}}-j \frac{Z}{z_{\mathrm{a}}} \sin k l_{\mathrm{d}}\right) \epsilon^{j k l} G_{\mathrm{M} 2}\right\} \frac{Z}{z_{\mathrm{s}}} G_{1}\right]
\end{aligned}
$$

$$
\beta=\frac{p_{\mathrm{Ms}}}{p_{1 \mathrm{~s}}}=1+\left(\cos k l_{\mathrm{d}}-j \frac{Z}{z_{\mathrm{a}}} \sin k l_{\mathrm{d}}\right) \epsilon^{j k l} G_{\mathrm{M} 2}
$$

In order that $\beta=0$

$$
G_{\mathrm{M} 2}=-\frac{\epsilon^{-j k l}}{\cos k l_{\mathrm{d}}-j \frac{Z}{z_{\mathrm{a}}} \sin k l_{\mathrm{d}}}
$$

and in order that $p_{1}=0$

$$
G_{1}=-\frac{1}{j \frac{Z}{z_{\mathrm{s}}} \sin k l}
$$

e) In case where the system is shown in Fig. 6 (e) $p_{\mathrm{M}}=0$ on the condition of $\beta=0$. Therefore this situation is meaningless.

\section{$4.3 m=1, n=2$}

In this case, Eqs. (22) and (23) are reduced to the following equations:

$$
\begin{gathered}
p_{2}=\left(A_{2}-\frac{B_{2}}{Z}\right) p_{\text {in }}-\left(A_{2}+\frac{B_{2}}{Z}\right) p_{\mathrm{r}}-G_{\mathrm{B}_{2}} p_{\mathrm{M}} \\
\left(\frac{Z}{z_{\mathrm{s}}}+\frac{Z}{z_{\mathrm{a}}}\right) p_{2}=\left(D_{2}-Z C_{2}\right) p_{\mathrm{in}}-\left(D_{2}+Z C_{2}\right) p_{\mathrm{r}}+G_{\mathrm{D}_{2}} p_{\mathrm{M}}
\end{gathered}
$$

f) In case where the system is shown in Fig. 6 (f) In order that $\beta=0$, Eq. (42) is reduced to the equation as follows:

$$
G_{2}=-E(k \Delta l) G_{1}
$$

where

$$
E(x)=\cos x+j\left(\frac{Z}{z_{\mathrm{s}}}+\frac{Z}{z_{\mathrm{a}}}\right) \sin x
$$

Also from Eq. (45), in order that $p_{2}=0, G_{1}$ must be represented by the equation as follows:

$$
G_{1}=\frac{1}{j \frac{Z}{z_{\mathrm{s}}} \sin k \Delta l\left[E\{k(l+\Delta l)\}+j \frac{Z}{z_{\mathrm{s}}} E(k \Delta l) \sin k l\right]}
$$

g) In case where the system is given in Fig. 6 (g) $p_{\mathrm{M}}$ is given as

$$
p_{\mathrm{M}}=\cos k l \cdot p_{2}+j Z \sin k l \cdot U_{2}
$$


hence

$$
\begin{aligned}
\frac{p_{2}}{p_{\text {in }}}=2 & \left(1+j \frac{Z}{z_{\mathrm{s}}} \sin k l \cdot G_{2}\right) \\
& /\left\{\left(1+j \frac{Z}{z_{\mathrm{s}}} \sin k l \cdot G_{2}\right) \Delta_{0}-\frac{Z}{z_{\mathrm{s}}} E(k l)\right. \\
& \left.\cdot\left[G_{1}+\left\{\epsilon^{j k\left(l+l_{\mathrm{d}}\right)}+j \frac{Z}{z_{\mathrm{s}}} \sin k\left(l+l_{\mathrm{d}}\right)\right\} G_{2}\right]\right\} \\
\frac{p_{\mathrm{Ms}}}{p_{\text {in }}}= & \frac{2 E(k l)}{\Delta_{0} \Delta} \cdot \frac{\Delta_{0}-\left(1+j \frac{Z}{z_{\mathrm{s}}} \sin k l \cdot G_{2}\right) \Delta}{1+j \frac{Z}{z_{\mathrm{s}}} \sin k l \cdot G_{2}}
\end{aligned}
$$

where

$$
\left.\begin{array}{l}
\Delta_{0}=\left(1+\frac{Z}{z_{\mathrm{s}}}+\frac{Z}{z_{\mathrm{a}}}\right) \epsilon^{j k\left(l+l_{\mathrm{d}}\right)}+\frac{Z}{z_{\mathrm{s}}} E(k l) \\
\Delta=\Delta_{0}-\frac{Z}{z_{\mathrm{s}}} E(k l) \frac{\left\{\epsilon^{j k\left(l+l_{\mathrm{d}}\right)}+j \frac{Z}{z_{\mathrm{s}}} \sin k\left(l+l_{\mathrm{d}}\right)\right\} G_{2}}{1+j \frac{Z}{z_{\mathrm{s}}} \sin k l \cdot G_{2}}
\end{array}\right\}
$$

In order that $\beta=0\left(p_{\mathrm{Ms}}=0\right)$

$$
G_{1}=-\left\{\epsilon^{j k\left(l+l_{\mathrm{d}}\right)}+j \frac{Z}{z_{\mathrm{s}}} \sin k\left(l+l_{\mathrm{d}}\right)-j \frac{\sin k l}{E(k l)} \Delta_{0}\right\} G_{2}
$$

In order that $p_{2}=0$

$$
G_{2}=-\frac{1}{j \frac{Z}{z_{\mathrm{a}}} \sin k l}
$$

h) In case where the system is shown in Fig. 6 (h) $p_{\mathrm{M}}$ is given as

$$
p_{\mathrm{M}}=\left(\cos k l_{\mathrm{d}}-j \frac{Z}{z_{\mathrm{a}}} \sin k l_{\mathrm{d}}\right) p_{2}
$$

In order that $\beta=0\left(p_{\mathrm{Ms}}=0\right)$

$$
p_{2 \mathrm{~s}}=0
$$

This situation is meaningless.

\section{4 $m+n \geq 4$}

Possible combinations of $G_{\mathrm{M} i}$ and $G_{i}$ are numberless in order that $\beta=0$ and $p_{n}=0$. But any other combinations are less advantageous compared with the case of $m+n=3$. If complexity is not considered, the acoustical feedback may be decreased by the two ways of insufficient conditions in which $\beta=0$, Eqs. (41) and (42).

\section{ELECTRICAL CONSTRUCTION OF $G_{i}$ AND CANCELING THE ACOUSTICAL FEEDBACK BY AN ELECTRICAL FEEDBACK}

\subsection{Electrical Construction of $G_{i}$}

$G_{i}$ is given by the ratio of $p_{\mathrm{s} i}$ to $p_{\mathrm{M}}$ and it contains the sensitivity of the microphone and the loudspeaker. Assume that a condenser microphone and a dynamic loudspeaker are used in this system. Figure 7 shows the block diagram of the control system, where

$K_{\mathrm{M}}$ : voltage sensitivity of microphone.

$\mu_{\mathrm{M}}$ : amplification factor of microphone amplifier.

$\mu_{\mathrm{e} i}$ : voltage amplification factor of the power amplifier for each loudspeaker, in which the suitable phase shift is contained.

$z_{\mathrm{eo}}$ : output impedance of the power amplifier.

$z_{\text {esi } i}$ : electrical impedance of each loudspeaker which contains the motional impedance

$I_{i}$ : electric current of each loudspeaker

$A_{\mathrm{s}}$ : force factor of loudspeaker

In Fig. 7

$$
G_{i} p_{\mathrm{M}}=p_{\mathrm{s} i}=\frac{A_{\mathrm{s}} I_{i}}{S_{\mathrm{s}}}=\frac{A_{\mathrm{s}}}{S_{\mathrm{s}}} \cdot \frac{K_{\mathrm{M}} \mu_{\mathrm{M}} \mu_{\mathrm{e} i}}{z_{\mathrm{eo}}+z_{\mathrm{es} i}} p_{\mathrm{M}}
$$

hence

$$
\mu_{\mathrm{e} i}=\frac{S_{\mathrm{s}}\left(z_{\mathrm{eo}}+z_{\mathrm{es} i}\right)}{A_{\mathrm{s}} K_{\mathrm{M}} \mu_{\mathrm{M}}} G_{i}
$$

Generally $z_{\mathrm{es} i}$ of each loudspeaker is not equal. But as the motional impedance is usually small compared with the pure electric impedance $z_{\mathrm{es}}, \mu_{\mathrm{e} i}$ can be denoted approximately as follows:

$$
\mu_{\mathrm{e} i} \fallingdotseq \frac{S_{\mathrm{s}}\left(z_{\mathrm{eo}}+z_{\mathrm{es}}\right)}{A_{\mathrm{s}} K_{\mathrm{M}} \mu_{\mathrm{M}}} G_{i}
$$

If the polarity of the loudspeaker is reversed, a negative sign must be added to $\mu_{\mathrm{e} i}$.

Figure 8 shows two examples of the control system. The characteristics required in electric circuits which should satisfy the equations, $\mu_{\mathrm{e} i}$ and $G_{\mathrm{M} 22}$, are not simple and it is difficult to realize the characteristics over the wide frequency range only by the

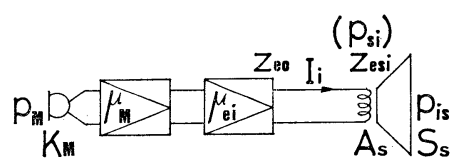

Fig. 7 Block diagram of control system. 


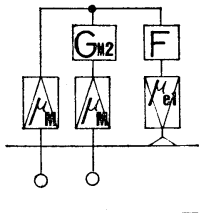

(a)

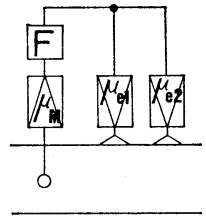

(b)
Fig. 8 Examples of control system.

analog technique. They require the digital technique.

5.2 Canceling the Acoustical Feedback by an Electrical Feedback

For the system shown in Fig. 6 (a), sound pressures and transfer functions in Fig. 5 are denoted as follows:

$$
p_{n}=p_{1}, \quad p_{n}=p_{10}, \quad p_{n \mathrm{~s}}=p_{1 \mathrm{~s}}
$$

and

$$
\left.\begin{array}{rl}
H_{1}=\frac{p_{10}}{p_{10}}=1, \quad H_{\mathrm{M}}= & \frac{p_{\mathrm{M}}}{p_{10}}=\cos k l \\
& +j\left(\frac{Z}{z_{\mathrm{s}}}+\frac{Z}{z_{\mathrm{a}}}\right) \sin k l \\
\beta=\frac{p_{\mathrm{Ms}}}{p_{1 \mathrm{~s}}}=\epsilon^{-j k l}, \quad \mu=\frac{p_{1 \mathrm{~s}}}{p_{\mathrm{M}}}=\frac{1}{1+\frac{z_{\mathrm{s}}}{Z}+\frac{z_{\mathrm{s}}}{z_{\mathrm{a}}}}
\end{array}\right\}
$$

and from equivalent circuit, Fig. 9, the relation between $p_{\mathrm{s} 1}$ and $p_{1 \mathrm{~s}}$ is described as

$$
p_{1 \mathrm{~s}}=\frac{1}{1+\frac{z_{\mathrm{s}}}{Z}+\frac{z_{\mathrm{s}}}{z_{\mathrm{a}}}} p_{\mathrm{s} 1}
$$

An electrical feedback loop is added to cancel the acoustical feedback as shown in Fig. 10. In which

$$
\begin{gathered}
\beta=\frac{p_{\mathrm{Ms}}}{p_{1 \mathrm{~s}}}=\frac{p_{\mathrm{Ms}}}{p_{\mathrm{s} 1}} \cdot \frac{p_{\mathrm{s} 1}}{p_{1 \mathrm{~s}}}=\frac{p_{\mathrm{Ms}}}{p_{\mathrm{s} 1}}\left(1+\frac{z_{\mathrm{s}}}{Z}+\frac{z_{\mathrm{s}}}{z_{\mathrm{a}}}\right) \\
G_{\beta}=-\frac{p_{\mathrm{Ms}}}{p_{1 \mathrm{~s}}}=-\frac{\epsilon^{-j k l}}{1+\frac{z_{\mathrm{s}}}{Z}+\frac{z_{\mathrm{s}}}{z_{\mathrm{a}}}}
\end{gathered}
$$

Figure 11 shows the electric system with feedback. Where $E_{\mathrm{M} 0}$ and $E_{\mathrm{Ms}}$ are the output of $\mu_{\mathrm{M}}$ caused by $p_{\mathrm{M} 0}$ and $p_{\mathrm{Ms}}$ respectively.

$$
\begin{gathered}
E_{\mathrm{Ms}}=K_{\mathrm{M}} \mu_{\mathrm{M}} p_{\mathrm{Ms}} \\
p_{\mathrm{s} 1}=\frac{A_{\mathrm{s}} I_{1}}{S_{\mathrm{s}}}
\end{gathered}
$$

Hence, the gain of the electrical feedback loop which cancels the acoustical feedback is

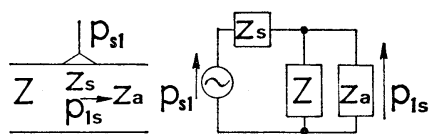

(a)

(b)

Fig. 9 Equivalent circuit for $p_{\mathrm{s} 1}$ and $p_{1 \mathrm{~s}}$, (a) cofiguration (b) its equivalent circuit.

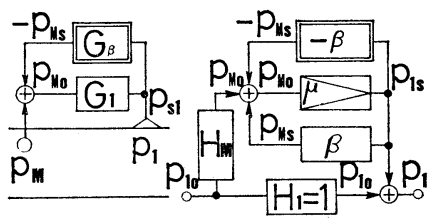

(a)

(b)

Fig. 10 Configuration of system in which an electrical feedback is added, (a) configuration (b) block diagram.

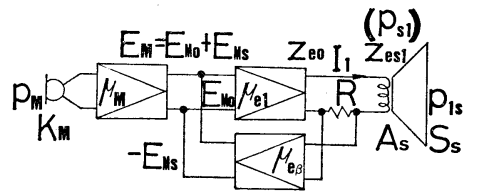

Fig. 11 Electric construction with a feedback loop.

$$
\begin{aligned}
\mu_{\mathrm{e} \beta} & =-\frac{E_{\mathrm{Ms}}}{I_{1} R}=-\frac{A_{\mathrm{s}} K_{\mathrm{M}} \mu_{\mathrm{M}}}{S_{\mathrm{s}} R} \cdot \frac{p_{\mathrm{Ms}}}{p_{1 \mathrm{~s}}} \\
& =-\frac{A_{\mathrm{s}} K_{\mathrm{M}} \mu_{\mathrm{M}}}{S_{\mathrm{s}} R} \frac{\epsilon^{-j k l}}{1+\frac{z_{\mathrm{s}}}{Z}+\frac{z_{\mathrm{s}}}{z_{\mathrm{a}}}}
\end{aligned}
$$

As the equation $p_{1}=0$ means that $p_{1_{\mathrm{s}}}=-p_{10}$, the gain of the power amplifire which cancels the noise under the condition of Eq. (99) is deduced as follows:

$$
\begin{aligned}
G_{1} & =\frac{p_{\mathrm{s} 1}}{p_{\mathrm{M} 0}}=\frac{p_{1 \mathrm{~s}}}{p_{\mathrm{M} 0}} \cdot \frac{p_{\mathrm{s} 1}}{p_{1 \mathrm{~s}}}=-\frac{p_{10}}{p_{\mathrm{M} 0}} \cdot \frac{p_{\mathrm{s} 1}}{p_{1 \mathrm{~s}}} \\
& =-\frac{1+\frac{z_{\mathrm{s}}}{Z}+\frac{z_{\mathrm{s}}}{z_{\mathrm{a}}}}{\cos k l+j\left(\frac{Z}{z_{\mathrm{s}}}+\frac{Z}{z_{\mathrm{a}}}\right) \sin k l}
\end{aligned}
$$

and by substituting Eq. (100) to Eqs. (90) and (91), the required amplification factor of the electric power amplifier is described as follows:

$$
\mu_{\mathrm{e} 1}=-\frac{S_{\mathrm{s}}\left(z_{\mathrm{e} 0}+R+z_{\mathrm{es} 1}\right)}{A_{\mathrm{s}} K_{\mathrm{M}} \mu_{\mathrm{M}}} \frac{1+\frac{z_{\mathrm{s}}}{Z}+\frac{z_{\mathrm{s}}}{z_{\mathrm{a}}}}{\cos k l+j\left(\frac{Z}{z_{\mathrm{s}}}+\frac{Z}{z_{\mathrm{a}}}\right) \sin k l}
$$




$$
\fallingdotseq-\frac{S_{\mathrm{s}}\left(z_{\mathrm{e} 0}+R+z_{\mathrm{es}}\right)}{A_{\mathrm{s}} K_{\mathrm{M}} \mu_{\mathrm{M}}} \frac{1+\frac{z_{\mathrm{s}}}{Z}+\frac{z_{\mathrm{s}}}{z_{\mathrm{a}}}}{\cos k l+j\left(\frac{Z}{z_{\mathrm{s}}}+\frac{Z}{z_{\mathrm{a}}}\right) \sin k l}
$$

By use of the electric system shown in Fig. 11, in which $\mu_{\mathrm{e} 1}$ and $\mu_{\mathrm{e} \beta}$ satisfy Eq. (101) or (102) and Eq. (99) respectively, a microphone and a loudspeaker system is able to control the noise in duct in avoidance of acoustical feedback. If the polarity of the loudspeaker is reversed, the minus sign in $\mu_{\mathrm{e} 1}$ and $\mu_{\mathrm{e} \beta}$ must be removed.

\section{CONCLUSION}

The principle of active control of noise in duct is explained and it is made clear in this paper what the pressure to be canceled out should be. And it is also made clear that canceling the original sound wave by the sound wave with the antiphase is equivalent to making the equivalent impedance of the active control system be zero, and there are three methods of picking up the canceling signal in active control.

We also showed the general equations of multimicrophone and multi-loudspeaker system and the conditions of control without acoustical feedback by means of impedance. Four suitable configurations and their conditions of control without acoustical feedback were deduced from the results of analysis. In addition, an electrical method which can cancel the acoustical feedback is proposed.

\section{REFERENCES}

1) P. Leug, "Process of silencing sound oscillation,"
U.S. Patent No. 2, 043, 416 (1934).

2) J. Okda, T. Nimura, and K. Kido, "Internal impedance of a system of electroacoustic transducers," J. Inst. Electron. Commun. Eng. Jpn. 44, 330-334 (1961) (in Japanese).

3) T. Kosaka and S. Yamada, "Active attenuation of repetitive low frequency noise in pipe," J. Acoust. Soc. Jpn. (J) 41, 316-321 (1985) (in Japanese).

4) J. Okda, Y. Nishimura, T. Usagawa, and M. Ebata, "Active control system by means of motional feedback," Tech. Paper 2nd Western/Pacific Regional Acoustics Conference (1985), pp. 482-486.

5) M. J. M. Jessel and G. A. Mangiante, "Active sound absorbers in duct," J. Sound Vib. 23, 383-390 (1972).

6) M. A. Swinbanks, "The active control of sound propagating in long duct," J. Sound Vib. 27, 411416 (1973).

7) G. Canevet and G. A. Mangiante, "Absorption acoustique active et anti-bruit a une-dimension," Acustica 30, 40-48 (1974).

8) A. A. Mazankov and V. V. Tyutekin, "Automonous active systems for the suppression of sound field in single-mode waveguide," Sov. Phys. Acoust. 22, 409-412 (1976).

9) J. H. B. Poole and H. G. Leventhall, "An experimental study of Swinbanks' method of active attenuation in duct," J. Sound Vib. 49, 257-266 (1976).

10) G. Canevet, "Active absorption in an air conditioning duct," J. Sound Vib. 58, 333-345 (1978).

11) Kh. Eghtesadi and H. G. Leventhall, "Active attenuation of noise: the Chelsea dipole," J. Sound Vib. 75, 127-134 (1981).

12) Kh. Eghtesadi and H. G. Leventhall, "A study of $n$ source active attenuator arrays for noise in duct," J. Sound Vib. 91, 11-19 (1983). 